\title{
First Experience with a New Portable Cardiopulmonary Bypass System - LIFEBRIDGE B2T with Percutaneous Femoral Cannulation
}

\author{
M Krane, D Mazzitelli, U Schreiber, A Mendoza Garzia, B Voss, \\ CC Badiu, R Lange, R Bauernschmitt
}

\begin{abstract}
Klinik für Herz- und Gefäßchirurgie, Deutsches Herzzentrum München des Freistaates Bayern, Klinik an der Technischen Universität München, München, Germany
\end{abstract}

\begin{abstract}
Cardiopulmonary bypass was rarely used for patients undergoing emergency circulatory resuscitation due to the large size and complicated set-up of currently available systems. The LIFEBRIDGE $B_{2}$ T System is a new portable system consisting of a disposable patient unit with a cardiopulmonary bypass circuit, a control unit, and a base unit with user interface. The portability of the system $(17 \mathrm{~kg}$, with a 2.5-hour LiPo battery capacity), as well as the simplicity of an automated user interface (including semi-automatic system priming and air elimination), is designed to address these concerns and allow for mobile mechanical support in a variety of situations and settings. In this first experience, we found the LIFEBRIDGE® $B_{2}$ T System to be safe and easy to use even for non-perfusionists and worthy of further investigation in non-standard surgical settings.
\end{abstract}

\section{Introduction}

Mechanical circulatory support (MCS) with cardiopulmonary bypass has been well established as the standard for maintaining cardiac and pulmonary function in patients undergoing cardiac surgery. Over the past decade, MCS has been utilized in various indications without cardiopulmonary bypass and has resulted in improved mortality, including bridge to transplant, bridge to recovery, cardiogenic shock and salvage in cardiopulmonary arrest. Despite these improvements, there has been little application for MCS in patients undergoing emergency circulatory resuscitation, most likely due to the cumbersome and complex systems that are currently available. Patients with medically refractory cardiogenic shock, pulmonary edema or those undergoing high risk percutaneous coronary interventions (PCI) are all at high risk of mortality and morbidity and may require emergency transport and/or circulatory resuscitation. Cardiogenic shock alone is associated with mortality greater than $60 \%$. LIFEBRIDGE $®$ Medizintechnik AG, Ampfing, Germany has developed a portable, modular, rapidly available "plug and play" mechanical circulatory support system called the LIFEBRIDGE $\mathrm{B}_{2} \mathrm{~T}{ }^{\circledR}$ System, which is designed to address the need for full MCS in mobile as well as in standard operative situations. The data included herein reflects our first experience utilizing the LIFEBRIDGE $B_{2} T \circledR$ System for circulatory support during beating heart coronary artery bypass surgery.

\subsection{System Description}

The LIFEBRIDGE $\mathrm{B}_{2} \mathrm{~T}{ }^{\circledR}$ System is a modular emergency system for cardiac and circulatory support characterized by the following features: modular configuration and semi-automatic priming, portability including integrated power supply offering up to two hours of stand-alone operation with LiPo batteries, and a seven stage air infusion prevention system which can tolerate massive venous ingress of air without jeopardizing patient safety.

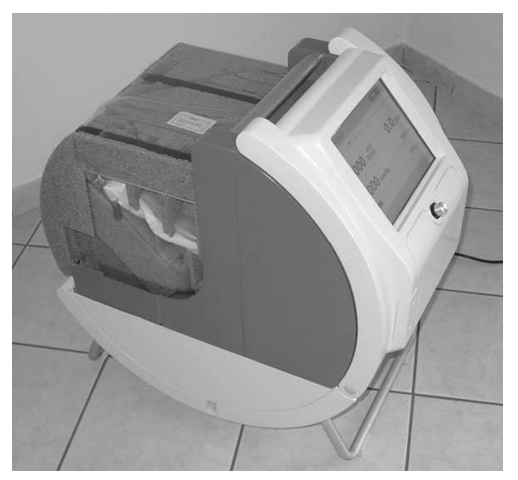

Fig. 1 The LIFEBRIDGE $B_{2}{ }^{\circledR}$ System 
The system is comprised of three modules: the Patient Module (PM), the Control Module (CM), and the Base Module (BM). The PM is the disposable part of the system. It represents a standardized extracorporeal circulation, consisting of a venous reservoir, a rotary blood pump, an oxygenator and an arterial filter. All components and the majority of tubing are fixed in molded parts made of expanded polypropylene. This provides excellent protection against external influences. Sensors used for pressure monitoring, flow level sensing are also integrated into the PM.

The CM contains all active parts necessary to drive and control the extracorporeal circulation. This includes the motor of the blood pump, a roller pump for air elimination and automatic clamps affecting the blood flow. Additional sensors for bubble detection and level sensing are incorporated to control system function and air elimination processes. The integrated backup battery allows stand-alone operation of the CM and PM for up to 30 minutes. In general, the $\mathrm{CM}$ is the non-disposable part of the system, excluding the user interface and the main power supply.

The BM, with its tubular frame, provides tilt-resistant standing of the LIFEBRIDGE system. It contains the main power supply and an embedded portable computer (EPC) with connected touch-sensitive flat panel and rotary switch, forming the user interface. In addition to the 30 minute battery capacity of the $\mathrm{CU}$, the BM also provides 2 additional hours of battery capacity, thus bringing the total capacity to 2.5 hours. Finally, the BM implements pivot mounting used to rotate the $\mathrm{CM}$ and PM by $90^{\circ}$ during the semi-automatic priming procedure, ensuring a secure elimination of air from the cardiopulmonary bypass circuit.

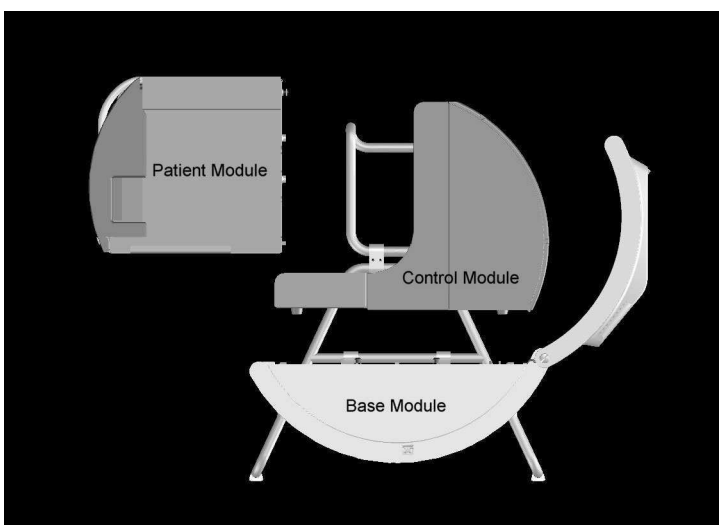

Fig. 2 Modular configuration of the LIFEBRIDGE® $\mathrm{B}_{2} \mathrm{~T}$ System
Extracorporeal circulation is achieved utilizing a closed system with arteriovenous connection to the patient. The LIFEBIDGE venous line is attached to the patient's blood vessels via a venous cannula. The centrifugal pump is preceded by a venous reservoir, intercepting any air intruding via the venous line. The blood pump is followed by an oxygenator and an arterial blood filter. Finally, the arterial line is attached to the patient via an arterial cannula. A bubble detector is placed right behind the arterial filter. Air detection at this point triggers the "Air Management" protocol. During this procedure the arterial line is closed first, with the arterial quick-closing clamp assuring short closing times of less than $300 \mathrm{~ms}$. By opening the shunt, the detected air is eliminated directly to the venous reservoir without jeopardizing the connected patient. When no more air is detected, the "Air Management" procedure concludes after a few seconds with the closing of the shunt and reopening of the arterial line. A schematic drawing of the extracorporeal circulation system is illustrated in figure 3 .

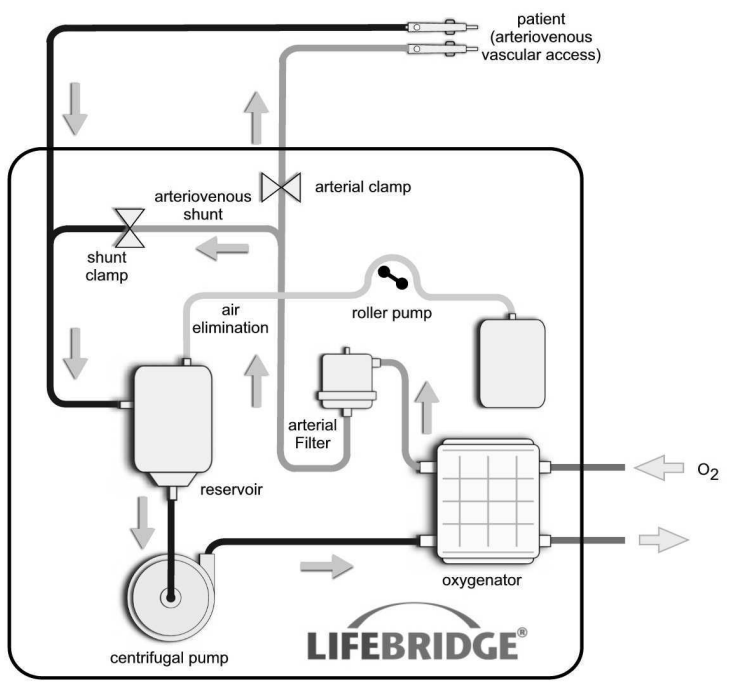

Fig. 3 Schematic drawing of extracorporeal circulation using the LIFEBRIDGE ${ } \mathrm{B}_{2}$ T System

The system for the prevention of air infusion is made up of seven stages with the following features:

- Air originating from the venous line or recirculated via the purge lines, is collected at the top of the reservoir by means of the buoy effect. This is also supported by the lower flow speed within the reservoir. A roller pump eliminates the air out of the closed system regardless of the actual pressure conditions.

- A >20 micron screen divides the reservoir into an entry and an exit volume, thus preventing a transition of micro-bubbles from the inlet to the outlet of the reservoir. 
- When a low-level condition in the venous reservoir is detected by the bottom level sensor, the blood pump is stopped.

- An important feature of centrifugal pumps is the inability to transport massive air. This safety aspect is reinforced by mounting the pumping head with its tangential outlet downside.

- The oxygenator itself is an air barrier. Via its recirculation line, air will be eliminated to the venous reservoir.

- The 40 micron arterial filter centers air by means of a forced circular flow. Via its purge line, air is led back to the venous reservoir.

\section{Methods}

The use of the Lifebridge $\mathrm{B}_{2} \mathrm{~T}$ for circulatory support was approved by the ethical committee of the Technische Universität München. We used the LIFEBRIDGE ${ }^{\circledR} \mathrm{B}_{2} \mathrm{~T}$ System in a patient who underwent beating heart coronary artery bypass surgery for complete revascularization. The patient was placed on mechanical circulatory support during the procedure with the LIFEBRIDGE ${ }^{\circledR} \quad \mathrm{B}_{2} \mathrm{~T}$ System due to hemodynamic instabilities at the beginning of off-pump coronary artery bypass surgery. Connection to the system was achieved via percutaneous cannulation of the femoral artery and the femoral vein utilizing a $24 \mathrm{~F}$ cannula (Edwards Lifesciences) for venous blood drainage and an $18 \mathrm{~F}$ cannula (Edwards Lifesciences) for arterial cannulation.

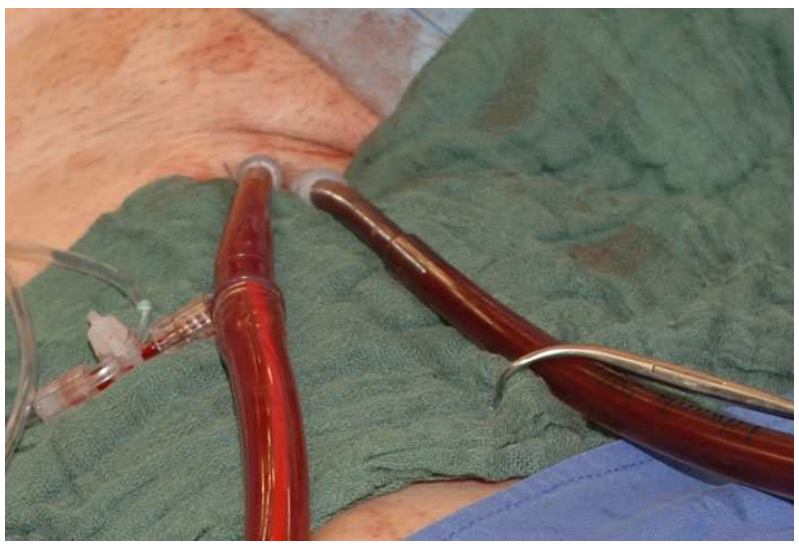

Fig. 4 percutaneous cannulation

Semi-automatic priming and automated air elimination were performed according to the manufacturer's Instructions for Use with no technical problems.

\section{Results}

The patient underwent successful beating heart coronary artery bypass surgery with mechanical circulatory support provided by the LIFEBRIDGE® $\mathrm{B}_{2} \mathrm{~T}$ System. Semi-automatic priming of the system was achieved in 5-10 minutes and the 7-step automated air elimination program prevented air embolism. No technical problems occurred during the use of the MCS system LIFEBRIDGE ${ }^{\circledR} \mathrm{B}_{2} \mathrm{~T}$. Cardiopulmonary bypass time was 135 minutes, with a target blood flow for the patient of $5.1 \mathrm{~L} / \mathrm{min}$. During circulatory support the system delivered a mean arterial blood flow of $4.4+0.42$ $\mathrm{L} / \mathrm{min}$ (range: $3.5-5.1 \mathrm{~L} / \mathrm{min}$ ).

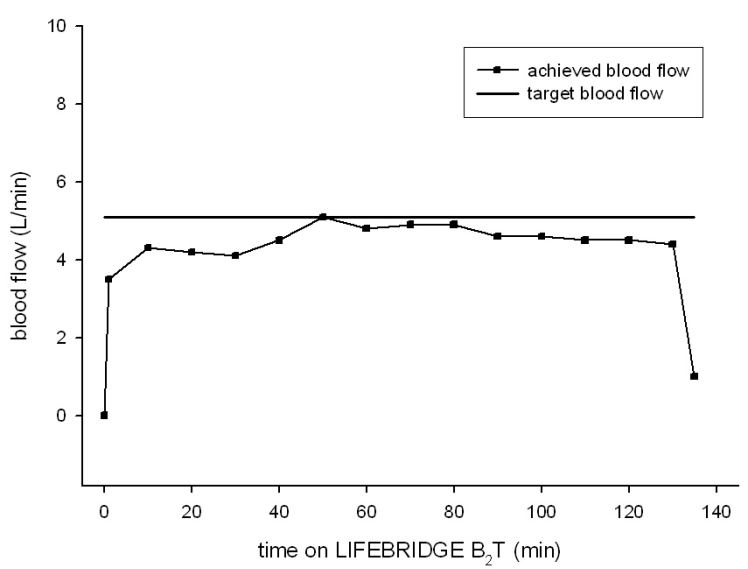

Fig. 4 blood flow during LIFEBRIDGE $\mathrm{B}_{2} \mathrm{~T}$ support

Mean negative pressure at the venous drainage was $94.4 \pm 30.4 \mathrm{mmHg}$ (range: $-45--126 \mathrm{mmHg}$ ) and mean arterial pressure during circulatory support was $68 \pm 6$ $\mathrm{mmHg}$ (range: $50-74 \mathrm{mmHg}$ ).

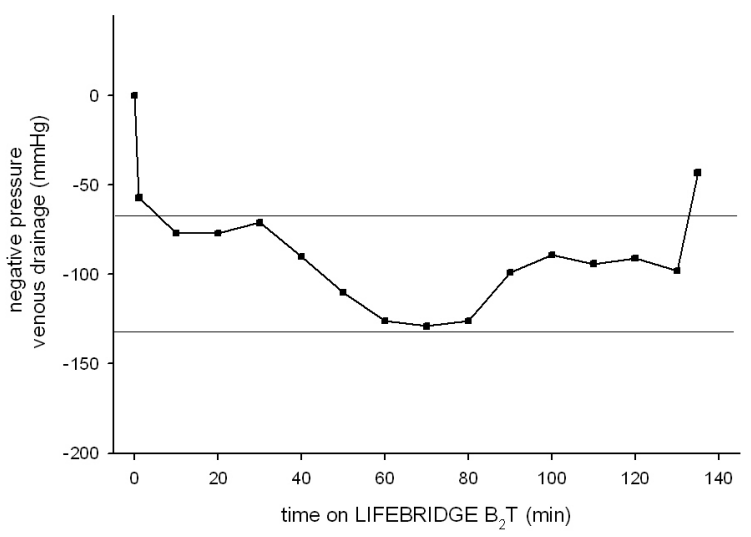

Fig. 5 negative pressure at the venous drainage during LIFEBRIDGE $\mathrm{B}_{2} \mathrm{~T}$ support. 


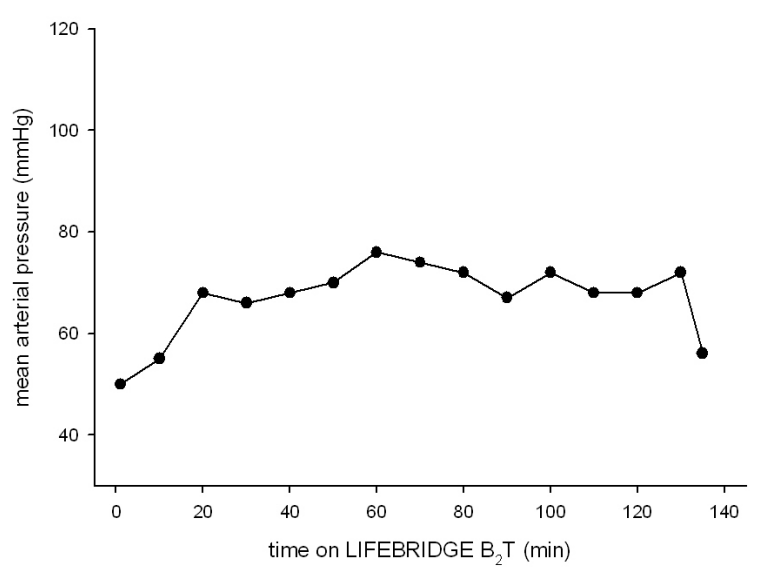

Fig. 6 mean arterial pressure during LIFEBRIDGE $\mathrm{B}_{2} \mathrm{~T}$ support

\section{Discussion and conclusions}

Early experience with the LIFEBRIDGE® $\mathrm{B}_{2} \mathrm{~T}$ System has shown it to be a feasible alternative to standard extracorporeal circulation in support of beating heart coronary artery bypass surgery, providing adequate arterial blood flow and an acceptable negative pressure at the venous cannula.

Percutaneous cannulation ( $18 \mathrm{~F}$ arterial/ $24 \mathrm{~F}$ venous) was performed without any complications. There were no signs of leg ischemia or bleeding complications during and after the operation. During the procedure the LIFEBRIDGE ${ }^{\circledR} \mathrm{B}_{2} \mathrm{~T}$ delivered a blood flow of $4.4 \pm 0.42$ $\mathrm{L} /$ minutes via percutaneous cannulation, which was close to the calculated blood flow of $5.1 \mathrm{~L} / \mathrm{min}$.

In the future, the system's semi-automatic priming system, automated air elimination protocol and lightweight portability will make the LIFEBRIDGE $®$ $B_{2} T$ System suitable for use during emergency transport as well as standard operating procedures. The "plug and play" aspects of the system make it easy to use for perfusionists and non-perfusionists alike. Further developments will be needed to automatically optimize clinically appropriate blood flows for individual patients. This could improve the safety of the system during emergency transports or temporary circulatory support during high risk PCI or beating heart surgery, especially when the system is used by non-perfusionists.

\section{Acknowledgements}

This work has been supported by an unrestricted educational grant from LIFEBRIDGE® Medizintechnik AG.

\section{References}

[1] Mehlhorn U, Brieske M, Fischer UM, Ferrari M, Brass P, Fischer JH, Zerkowski HR. LIFEBRIDGE: A

portable, modular, rapidly available "plug-and-play" mechanical circulatory support system. Ann Thorac Surg.2005; 80: 1887-92.

[2] Krane M, Bauernschmitt R, Lange R. Die HerzLungen-Maschine. In: Wintermantel E (Hrsg.) Medizintechnik Life Science Engineering. Springer, Berlin Heidelberg, 1069-1082.

[3] Ferrari M, Figulla HR. Circulatory assist devices in cardiology. Dtsch Med Wochenschr. 2005; 130: 652-6.

[4] Madershahian N, Nagib R, Wippermann J, Strauch $\mathrm{J}$, Wahlers T. A simple technique of distal limb perfusion during prolonged femoro-femoral cannulation. J Card Surg. 2006; 21: 168-9.

Address for correspondence

Markus Krane, M.D.

Klinik für Herz- und Gefäßchirurgie

Deutsches Herzzentrum München des Freistaates Bayern Klinik an der Technischen Universität München

Lazarettstraße 36

80636 München

krane@dhm.mhn.de 